\title{
Comparação entre duas estratégias para a detecção precoce do hipotiroidismo congênito
}

\author{
L.S. Ward, *R.M.B. Maciel, R.F. Magalhães, *I.S. Kunil, *G.K. Kurazawa, *L.K. Matsumura, \\ *J.G.H. VIEIRA
}

Disciplina de Medicina Interna, Departamento de Clínica Médica e Centro de Assistência Integrada à Saúde da Mulher (CAISM), F aculdade de Ciências Médicas da Universidade Estadual de Campinas, Campinas, SP. *Disciplina de Endocrinol ogia, Departamento de Medicina, Universidade Federal de São Paulo - Escola Paulista de Medicina, São Paulo, SP.

\begin{abstract}
RESUMO - Oвj etivo. Comparar em recém-nascidos ( $R N$ ) duas estratégias diferentes para o rastreamento do hipotiroidismo congênito (HC), a dosagem primária de TSH no sangue colhi do do cordão umbilical (método 1) e a dosagem primária de T4 no sangue colhido por punção de calcanhar no $2^{\circ}$ dia de internação (método 2).

MÉ Todos. Os autor es compararam as duas estratégias em 10.000 RN. Dosaram o TSH por método imunofluorimétrico sensível em papel de filtro e o T4 por radioimunoensaio em papel de filtro. A coleta de sangue do calcanhar foi realizada no $2^{\circ}$ dia de vida
\end{abstract}

Resultados. Os dois programas diagnosticaram todos os casos de HC nos RN (4 casos, 1/2.500 R N). $O$ índice de rechamada por coleta inadequada foi

\section{INTRODUÇÃO}

O hipotiroidismo congênito $(\mathrm{HC})$ é um distúrbio metabólico sistêmico caracterizado pela deficiência da produção dos hormônios tiroidianos ${ }^{1}$. Problemas na ontogênese do sistema hipotálamo-hipófise-tirói de podem gerar uma grande variedade de al terações anatômi cas ou funcionai s causadoras do $H C^{2}$. A doença tem uma incidência relativamente alta, de cerca de $1 / 4.000$ recém-nascidos (RN) vivos ${ }^{1-5}$. Em zonas iodoprivas, essa incidência chega a 1/ $2.000 \mathrm{RN}^{6}$; isto é particularmente importante para o Brasil, que ainda possui extensas áreas com graus variáveis de deficiência de iodo.

Ao nascimento, os sinais sugestivos de HC são escassos na absoluta maioria dos casos, extremamente inespecíficos e vagos, raramente despertando a atenção do neonatologista ${ }^{1-3}$. E m países europeus, onde os RN ficam internados até os cinco dias de vida, estudo epidemiológi co realizado antes da instalação dos programas de detecção precoce do HC demonstrou que menos do que $5 \%$ dos casos eram diagnosticados clinicamente por ocasião da internação no berçário; este trabal ho também evi- nulo no método 1 e de $8,5 \%$ ( 850 R N) no método 2.0 índice de reconvocação para confirmação de resultados foi de $0,06 \%$ (6 R N) no método 1 e de $2,25 \%$ (225 RN) no método 2; quando este método incluía também a dosagem suplementar de TSH, o índice baixou para 1,63\% (163 RN).

Conclusão. Os dados dos autores evidenciam a superioridade técnica da coleta de sangue a partir do cordão umbilical em relação à punção de calcanhar, assim como da dosagem primária de TSH em relação à de T4, uma vez que apresentam índices muito menores de reconvocação.

UNITERMOS: Rastreamento. Hipotiroidismo congênito. TSH. T4.

denciou que apenas $10 \%$ das crianças portadoras de HC tiveram suspeita clínica até o 1으ês, 35\% até o 3ํㅡês e $70 \%$ durante todo o 1ㅇ ano de vida ${ }^{7}$. De maneira semel hante, apenas 3\% das crianças diagnosticadas no programa norte-americano de triagem do HC tinham tido suspeita clínica por ocasião do nascimento ${ }^{8}$. E m nosso meio, onde a al ta é dada precocemente, muitas vezes antes de o RN completar 24 horas de vida, o diagnóstico no berçário é praticamente impossível ${ }^{9}$.

Os hormônios tiroidianos são essenciais para a maturação cerebral ${ }^{10}$. Esses efeitos metabólicos são críticos no desenvolvimento do tecido nervoso no período pré-natal e pós-natal. As crianças com HC são protegi das dos efeitos del etérios da fal ta dos hormônios tiroidianos durante o período gestacional pela passagem transplacentária de T4 da mãe para o feto ${ }^{11}$. A partir do nascimento, porém, essa proteção desaparece, e aqueles RN tratados após o 3 mês de vida apresentarão, geralmente, retardo físi co e mental ${ }^{1-5}$. A análise de dados anteriores ao estabelecimento dos programas de rastreamento, proveni entes de crianças portadoras de HC não tratadas antes dos três meses de idade, 
indi ca que $20 \%$ terão coeficiente de intel igência (QI) normal, $25 \%$ terão retardo mental leve, 35\% serão moderadamente afetados e $20 \%$ terão retardo mental severo $(\mathrm{QI}<50) ; 60 \%$ terão ataxia cerebelar ${ }^{7,12}$. Esses dados indicam que os casos normais e levemente afetados poderiam se integrar à população normal, não necessitando alterar de forma mais significante seu modo de vida; entretanto, os 55\% dos RN restantes exigiriam institucionalização ou internação. Esses pacientes representam um custo considerável para toda a sociedade ${ }^{13}$.

Desde que o diagnóstico clínico do HC é difícil e incomum antes do 3o mês de vida e que a introdução de terapêutica substitutiva precoce é vital para se evitar as mani festações da doença e suas seqüel as, a solução é um programa de detecção precoce do HC realizado durante a primeira semana de vida em todas as crianças nasci das vivas. A partir do desenvolvimento de métodos de radiomunoensaio para dosar a tiroxina total (T4) em eluatos de sangue total seco col hidos em papel de filtro, seguidos das determinações da tirotrofina (TSH, do inglês thyroid stimulating hormone), vários programas de rastreamento para a detecção precoce de HC foram implantados e existem com uma rotina bastante bem estruturada em vári os lugares do mundo (EUA, Canadá, Europa, Austrália eJ apão) $)^{3,5}$. Em estudos relativos ao custo-benefício dos programas de detecção precoce de hipotiroidismo, nós demonstramos no Brasil, à semel hança do que ocorre em outros países, que a criação desses programas é altamente benéfica ${ }^{13}$.

Existem, em nosso meio, duas estratégias principais utilizadas para o diagnóstico precoce do hipotiroidismo: o programa recomendado pela Comissão de Hipotiroi dismo Congênito da Sociedade Brasileira de Endocrinologia e Metabologia, que utiliza a dosagem primária de TSH no cordão umbi lical, tendo em vista a alta precoce da maioria dos $\mathrm{RN}$, reservando a dosagem de T4 na mesma amostra para exame confirmatório ${ }^{14}$; e o programa recomendado pela Associação de Pais e Amigos dos Excepcionais de São Paulo (APAE), que emprega coleta por punção de calcanhar na alta do RN da maternidade com a dosagem primária de T4, segui$\mathrm{da}$, atualmente, da dosagem de TSH na mesma amostra como segundo exame ${ }^{15}$. Ainda existem controvérsias quanto à melhor estratégia a ser utilizada em nosso país.

I mplantamos o programa de rastreamento para a detecção precoce do HC na maternidade do Centro de Assistência Integrada à Saúde da Mulher (CAISM) da Universidade Estadual de Campinas (UNICAMP) em outubro de 1989 utilizando a dosagem primária de TSH realizado pela E scola Paulis- ta de Medicina, U niversidade F ederal de São Paulo (EPM), em sangue de cordão, segundo o programa recomendado pela Sociedade Brasileira de Endocrinologia e Metabologia (método 1 ). Em fevereiro de 1991, por determinação da Secretaria de Saúde do Estado de São Paulo, esta maternidade passou a colher, simultaneamente, sangue de calcanhar, por ocasi ão da alta no $2^{\circ}$ dia de internação, para a dosagem de T4 pela APAE (método 2). Assim, de fevereiro de 1991 até outubro de 1994, essa maternidade realizou os dois procedimentos simultaneamente, o que nos permitiu comparar, de maneira ímpar na literatura, as duas metodologias num grande número de casos.

\section{MATERIAIS E MÉTODOS}

\section{Considerações metodológicas: T4 X TSH}

Muitos programas de rastreamento para o diagnóstico precoce do $\mathrm{HC}$, especialmente nos Estados Unidos, começaram utilizando a dosagem do T4 como teste inicial, seguida sempre da determinação de TSH confirmatória nos $20 \%$ de RN que apresentavam os menores valores de T $4^{8}$. No início dos programas, a vantagem do T4 era decorrente de sua execução mais fácil, uma vez que o ensaio era metodologicamente mais simples; hojeem dia, questiona-se essa vantagem, pois os ensaios de TSH são também simples e rápidos. A principal desvantagem do uso do T4 é o grande número de casos falsos-positivos, tendo em vista as diversas condições em que há diminuição transitória do T4 no RN, tais como prematuridade, baixo peso e uma série de outros distúrbios neonatais; além disso, também apresentam val ores baixos de T4 OS RN com diminuição da proteína ligadora da tiroxina (TBG, do inglês thyroxine binding globulin), presentes em 1 para cada 9.000 nascimentos ${ }^{16}$. Outra desvantagem importante do emprego do T4 em programas de rastreamento para o diagnóstico precoce do HC é a obtenção de casos falsos-negativos, quando se encontram RN com HC apresentando 4 normal, tendo em vista a passagem transplacentária de T4 da mãe para o feto ${ }^{11}$.

A dosagem primária de TSH, com dosagem suplementar do T4, nos casos em que os valores de TSH encontrados são superiores aos valores normais para a faixa etária, é mais específica, discriminando perfeitamente os RN normais dos portadores de HC primário. Mas também não é procedimento isento defal has, pois deixa de diagnosticar os raros portadores de hipotiroi dismo secundário e terciário, originando resultados falsos-negativos; a atenuante é que a dosagem primária de T4 também pode deixar de diagnosticar essa eventualidade ${ }^{17}$. 
Além disso, nesses casos, o quadro clínico é geralmente mais rico, devido à associação do hipoti roidismo com outras deficiências hipofisárias e/ou hipotalâmicas, facilitando o diagnóstico clínico. Com o advento dos programas de rastreamento para o diagnóstico precoce do HC, observou-se uma série de disfunções tiroidianas próprias do período neonatal que evoluem com TSH el evado e que causam resultados falsos-positivos de hipotiroidis$\mathrm{mo}^{18}$. A dosagem suplementar de T4 evita que tais pacientes sejam considerados portadores de $\mathrm{HC}$, pois o T4, nesses casos, situa-se dentro da faixa de normalidade.

\section{Consi derações metodol ógi cas: cordão x cal canhar}

A col eta de sangue de cordão apresenta a vantagem de não alterar a rotina de maternidade que dão alta precoce ao RN de parto normal, de não elevar os custos pela manutenção hospitalar da mãe e do RN, e de não expor os RN a risco de infecção hospitalar pela permanência prolongada no berçário. A desvantagem de seu emprego é a impossibilidade de obtenção concomitante do material de pesquisa da fenilcetonúria (PKU), cuja coleta deve ser realizada após um determinado número de alimentações protéicas, o que acontece habitualmente a partir do 3o dia de vida ${ }^{19,20}$.

A col eta obti da por punção de cal canhar entre o 3o e 5o dia de vida permite a pesquisa de PKU na mesma amostra. Apresenta como desvantagem, porém, a permanência do RN e das mães por tempo prolongado no hospital, o que geraria no Brasil uma dificuldade para a implantação do programa, tendo em vista a alta precoce generalizada que ocorre em todas as maternidades. Adicionalmente, os problemas técnicos da col eta são maiores devido à menor quantidade de sangue col hido e à necessidade de pessoal e material especializados ${ }^{1,21}$.

\section{CASUÍSTICA}

Analisamos 10.000 RN vivos na maternidade do CAISM/UNICAMP, no período de fevereiro de 1991 a fevereiro de 1994, o que representa a quase totalidade dos partos feitos nesse período. Essas crianças foram submeti das, concomitantemente, ao programa de rastreamento para o diagnósti co precoce do HC utilizando a dosagem de TSH em sangue de cordão e à dosagem de T4 em sangue do calcanhar no $2^{\circ}$ dia. A seguir, confrontamos os índices de rechamada dos dois programas por meio dos registros do Serviço Social do CAISM, que rel aciona todos os RN reconvocados; estes tiveram seus prontuários revistos para se estabelecer o motivo da reconvocação, os resultados dos novos exames laborato- riais e o seguimento pediátrico para definição do seu estado tiroidiano.

\section{MÉTODOS}

O procedimento para a dosagem do TSH em sangue de cordão (método 1) baseou-se na coleta, em sala de parto, de sangue de cordão umbilical. $\mathrm{Na}$ rotina do serviço de sala de parto da maternidade do CAISM já ocorria a coleta de material de sangue de cordão para a tipagem sanguínea e outros exames; desta maneira, não houve al teração da rotina, apenas pingando-se mais al gumas gotas de sangue diretamente do cordão umbilical sobre o papel de filtro para a dosagem do TSH. A pós a secagem do papel, este era guardado em saco plástico para ser enviado por via postal ao L aboratório do Centro de Detecção Precoce de Hipotiroidismo Congênito da Disciplina de Endocrinologia da Escola Paulista de Medicina, Universidade Federal de São Paulo (EPM-UNIFESP), para a dosagem do TSH.

O procedimento para a dosagem de T4 em sangue do calcanhar (método 2) envolveu a punção com lanceta do calcanhar do RN no último dia de internação (habitual mente 2으 dia de vida) por pessoal da equipe de enfermagem e coleta no papel de filtro. A pós a secagem à temperatura ambiente, o papel era enviado ao Laboratório da APAE, em São Paulo, para a dosagem do T4.

O TSH e O T4 São dosados na EPM-UNIFESP por mei o de métodos imunofluorimétricos; para a dosagem de TSH em papel de fil tro, usa-se um anticorpo monoclonal de captura ligado em fase sól ida e um anticorpo monoclonal de reconhecimento ${ }^{22}$. Esse método possui sensibilidade de $0,1 \mathrm{mu} / \mathrm{L}$ e coeficientes de variação intra e interensaio de $5 \%$ e $10 \%$, respectivamente. Consideramos como nível de corte $20 \mathrm{mu} / \mathrm{L}$, a partir do qual a amostra é considerada suspeita. O T4 apresenta sensibilidade de $1,6 \mathrm{mg} / \mathrm{dL}$ e coeficiente de variação intra e interensaio de $5 \%$ e $10 \%$, respectivamente.

O T4 é dosado na APAE por um radioi munoensaio com sensibilidade de $1 \mathrm{mg} / \mathrm{dL}$ e coefi cientes de variação intra e interensaio de $6 \%$. O valor de corte considerado pela APAE é de $6 \mathrm{mg} / \mathrm{dL}$; desde junho de 1993, a APAE vem acrescentando, também, a medida do TSH por meio de um método comercial imunofluorimétrico, similar ao da EPM-UNIFESP, aqueles casos de RN que se apresentem no percentil 10 inferior do ensaio do $T 4^{15}$.

\section{RESULTADOS}

O período de implantação do programa de rastreamento para o diagnóstico precoce de $\mathrm{HC}$ no 
CAISM-UNICAMP utilizando o método 1 durou cerca de dez dias, a partir do que nenhum RN deixou de ter o material col hido corretamente identificado e enviado; como já mencionado, o CAISM-UNICAMP já possuía uma rotina de coleta de sangue umbilical para tipagem sanguínea e reações sorológicas para lues. Por outro lado, o tempo de implantação do programa de rastreamento para o diagnóstico precoce do HC empregando o método 2 envolveu um período de treinamento mais longo, de cerca de três meses, já que a punção de calcanhar não era rotineiramente efetuada na neonatologia e obrigou o treinamento de pessoal.

Suspeitou-se do diagnóstico de HC em quatro RN em ambos os programas, o que representa um caso para cada 2.500 RN vivos. Confirmou-se o diagnóstico em todos mediante dosagem de TSH e de T4 livre em sangue venoso.

Comparando-se os resultados dos 10.000 RN vivos submetidos a ambos os métodos de rastreamento, observamos que o índice de rechamada devido à coleta inadequada de material para as dosagens hormonais foi nulo no método 1 (TSH no cordão), enquanto que foi de $8,5 \%$ ( $850 \mathrm{RN}$ ) pel o método 2 (T4 no calcanhar). O principal problema que originou a reconvocação pela APAE foi o material escasso obtido na col heita de sangue do cal canhar.

O índice de reconvocação para confirmação de resultados foi de $0,06 \%$ (6 RN) no método 1 e de 2,25\% (225 RN) no método 2, e com o emprego da dosagem de TSH como segundo exame, esse índice passou a ser de 1,63\% (163 RN) (ver tabela).

\section{DISCUSSÃO}

A prevenção de um caso de retardo mental decorrente de hipotiroidismo congênito representa benefíci o incalculável para o próprio indivíduo, sua família, assim como para a sociedade, que deixará de arcar com uma pessoa improdutiva, que necessitaria de cuidados especiais por toda vida. Embora social, e mesmo moralmente, pareça imperativo prevenir tal doença, cuja inci dência é relativamente el evada e que potencial mente pode ser curada, a pouca disponibilidade de recursos econômicos alocados por área de saúde em nosso país tornou necessária a demonstração de que, também do ponto de vista econômico, é mais conveniente prevenir do que tratar um caso de cretinismo ${ }^{13}$. Programas de rastreamento para a detecção precoce de HC são essenciais para a identificação da doença, cuja freqüência e pouca sintomatologia no RN obrigam a atenção especial por parte de organismos de saúde pública ${ }^{21,23}$. No Brasil, a detecção de HC é obrigatória por lei desde 1985. No entanto, apenas o Estado

\begin{tabular}{|c|c|c|}
\hline Índice de rechamada (\%) & Método 2 & Método 1 \\
\hline por coleta inadequada & $\begin{array}{c}8,5 \% \\
(850 \mathrm{RN})\end{array}$ & $\begin{array}{c}0 \% \\
(0 \mathrm{RN})\end{array}$ \\
\hline para confirmação de resultados & $\begin{array}{c}2,25 \% \\
(225 \mathrm{RN})\end{array}$ & $\begin{array}{l}0,06 \% \\
(6 \mathrm{RN})\end{array}$ \\
\hline $\begin{array}{l}\text { para confirmação com } \\
\text { TSH como } 2^{\circ} \text { exame }\end{array}$ & $\begin{array}{c}1,63 \% \\
(163 \mathrm{RN})\end{array}$ & - \\
\hline
\end{tabular}

do Rio de J anei ro possui normatização a respeito e ainda existem controvérsias quanto à melhor estratégia a ser utilizada em programas de Saúde Pública no Brasil.

Os dois programas mais utilizados no país foram simultaneamente executados na UNICAMP por período de três anos. Utilizamos esse fato para efetuar uma comparação entre os métodos. Nossos dados mostraram incidência de HC de 1/2.500 RN nesse período, o que representa um número superior ao descrito em áreas não carentes de iodo. Acreditamos que tal fato possa decorrer de nosso serviço atender a muitas pacientes provenientes de regi ões sabidamente carentes em iodo, como Minas Gerais, Goiás e Mato Grosso do Sul.

Os resultados obtidos mostram que a coleta de sangue de cordão umbilical, quando comparada à punção do cal canhar, não obriga a rechamada, uma vez que o material é abundante e de fácil manipulação; o número de rechamadas pelo método 2 é muito al to para um programa que pretende atingir todos os RN do Brasil. O método 1 também tem a vantagem de não interferir na rotina hospitalar, permitindo a alta precoce e prevenindo a possibilidade de infecção, pel o mai or tempo de permanência que o RN teria no berçário, bem como reduz os custos de internações. Entretanto, não permite a pesquisa de PKU na mesma amostra; esta, entretanto, pode ser realizada por ocasião da primeira visita do RN ao Posto de Saúde ou ao pediatra ${ }^{14}$.

A punção de calcanhar, ao contrário, fornece material escasso, aliado à dificuldade de col eta inerente ao fato de tratar-se de criança recém-nascida e das dificuldades em treinar adequadamente pessoal para sua realização. Além disso, obriga a permanência da mãe e do RN por mais tempo na maternidade, pelo menos por dois dias, trazendo outras conseqüências, como o aumento do risco de infecção e a el evação dos custos hospitalares.

A comparação entre os dois programas em relação à estratégia utilizada mostrou que a dosagem 
primária de T4 condicionou um número muito mai or de reconvocações, em comparação com a dosagem primária de TSH ( $225 \times 6)$, decorrente da considerável quantidade de falsos-positivos que o T4 apresenta, pois é passível de reduções transitórias de di versas causas, entre as quais se destacam a prematuridade, o baixo peso e patol ogias neonatais relativamente freqüentes em nosso meio. $\mathrm{O}$ uso da dosagem complementar de TSH diminuiu o índice de rechamada para confirmação de diagnóstico pelo método 2 (163 RN), embora ainda tenha permanecido mais elevado do que no programa pel o método 1 (6 RN) que utilizou oTSH como exame primário.

Além do transtorno, do trauma, dos problemas psicossociais e econômicos que a convocação para confir mação do diagnósti co obviamente causa à família, mobilizada pela possibilidade de o RN poder vir a apresentar deficiência mental, a rechamada representa um ônus significativo ao sistema de saúde pública. Além disso, muitos RN desse enorme contingente de reconvocados não respondem à chamada, quer por dificuldade de localização, quer pel a ignorância dos riscos por parte da mãe; tendo em vista o grande número de RN que não atendem à reconvocação, não existe um esforço concentrado por parte dos responsáveis dos programas para a localização de todos os suspeitos, com a conseqüente perda dos reais casos de HC que poderiam ter sido diagnosticados.

Nossos resultados evidenciam a superioridade da técni ca de col eta por punção de cor dão umbilical e do método de dosagem de TSH primário com T4 complementar (método 1) no rastreamento do hi potiroidismo primário. Concluímos que tal estratégia, al ém de evitar um grande número de convocações onerosas e desnecessárias para confirmação de resultado, tem melhor relação custo-benefício e evita os problemas de coleta inadequada de material por punção de calcanhar.

\section{AGRADE CIMENTOS}

Este trabalho foi realizado com o apoio financeiro do Fundo de Incentivo à Pesquisa do Banco do Brasil (FIPEC), projeto 1037, Consel ho Nacional de Desenvolvimento Científico e Tecnológico (CNPq), projeto 405494/88.2, Agência Internacional de Energia Atômica, projeto 302-E1-BRA-5318.1, e Centro de Estudos de Endocrinologia da Escola Paulista de Medicina (CENEPAM). Os autores agradecem o empenho das enfermeiras, assistentes administrativas e sociais do CAISM, assim como à assistência administrativa de J oão L. Movio e Alzira M. Possedente, da EPM-UNIFESP.

\section{SUMMARY}

\section{Comparison between two strategies for the precocious detection of congenital hypothy- roidism}

Oвj ective. Compare two different strategies in newborn screening for congenital hypothyroidism, primary TSH in the umbilical cord blood (method 1 ) and primary $\mathrm{T} 4 \mathrm{in}$ blood collected from the heel in the 2 nd day of life (method 2).

Methods. We compared both strategies in 10,000 newborns, measuring TSH by a sensitive immunofluorimetric assay and $\mathrm{T} 4$ by a radi oi mmunoassay.

Results. Both strategies detected all cases of hypothyroidism (4 cases, 1/ 2,500 newborns). The recalling index owing to insufficient amount of blood to perform the assays was zero in method 1 and $8.5 \%$ (850 newborns) in method 2. The recalling index for confirmation of the results was $0.06 \%$ (6 newborns) in method 1 and 2.25\% (225 newborns) in method 2; when method 2 included supplementary TSH, the recalling index was reduced to $1.63 \%$ (163 newborns).

Conclusion. Our data indicate the technical superiority of the umbilical cord blood compared to heel and primary TSH compared to primary T4 in the neonatal thyroid screening for congenital hypothyroi dism. [Rev Ass Med Brasil 1998; 44 (2): 81-6.]

KEY WORDS: Screening. Congenital hypothyroidism. TSH. T4.

\section{REFERÊNCIAS BIBLIOGRÁFICAS}

1. Vassart G, Dumont JE, Refetoff S. Thyroid disorders. In Scriver CR, Beaudet AL, Sly WS, Valle D (eds): Metabolic basis of inherited diseases, $5^{\text {th }}$ ed. New York, McGraw-Hill, 1995; 2.883-928.

2. Burrow GA, Fisher DA, Larsen PR. Maternal and fetal thyroid function. N Engl J Med 1994; 331: 1.072-8.

3. LaFranchi S. Congenital hypothyroidism: a newborn screening success story? Endocrinologist 1994; 4: 477-86.

4. New England Congenital Hypothyroidism Collaborative. Neonatal hypothyroidism screening: status of patients at 6 years of age. J Pediatr 1985; 107: 915-9.

5. Dussault J H. An update on screening for congenital hypothyroidism. Thyroid Today 1985: 8: 1-6.

6. Delange F, Thilly C, Bourdoux P, Ermans AM. Occurrence and significance of disorders of thyroid function during the neonatal period in endemic goiter areas. In Medeiros-Neto G, Maciel RMB, Halpern A (eds): I odine deficiency disorders and congenital hypothyroidism. São Paulo, Aché, 1986; 218-21.

7. J acobsen BB. Congenital hypothyroidism: pre-screening experience. In Dussault J H, Walker P (eds): Congenital hypothyroidism. New York, Marcel Dekker, 1983; 147-50.

8. Fisher DA, Dussault J H, Foley J r TP et al. Screening for congenital hypothyroidism: results of screening one million North American infants. J Pediatr 1979; 94: 700-8. 
9. Maciel RMB. Neonatal thyroid screening in Brazil: the pilot experience of Escola Paulista de Medicina. In Schmidt BJ , Diament AJ, Loghin-Grosso NS (eds): Current trends in infant screening. Amsterdam, Exerpta Medica, 1989; 117-20.

10. Poterfield SP, Hendrich CE. The role of thyroid hormones in prenatal and neonatal neurological development - current perspectives. Endocrinol Rev 1993; 14: 94-104.

11. Vulsma T, Gons MH, de Vijlder J . Maternal-fetal transfer of thyroxine in congenital hypothyroidism due to a total organification defect of thyroid agenesis. N Engl J Med 1989; 321: 13-6.

12. Klein AH, Meltzer S, Kenny F. Improved prognosis in congenital hypothyroidism treated before age three months. J Pediatr 1972; 81: 912-7.

13. Ward LS, Zanella MT, Menabó E et al. Estimativa da relação custo-benefício de um programa de detecção precoce de hipotiroidismo congênito. Rev Ass Med Brasil 1988; 34: 16-10.

14. Meirelles RMR, Maciel RMB, Machado FA, Castro AS. Subsídios para a regulamentação das leis que estabel ecem a obrigatoriedade do diagnóstico do hipotiroidismo congênito e fenilcetonúria. Arq Bras Endocrinol Metab 1991; 35: 12-4.

15. Coelho-N eto J R. Resultados do rastreamento do hipotiroidismo congênito da APAE-SP, VI Encontro Brasileiro de Tiróide, Campinas, 1995.

16. Fisher DA. Euthyroid low thyroxine (T4) and triiodothyronine (T3) states in prematures and sick neonates. Pediatr
Clin North Am 1990; 37: 1.297-312.

17. Hanna CE, Krainz PL, Skeels MR et al. Detection of congenital hypopituitary hypothyroidism: ten-year experience in the Northwest Regional Screening Program. J Pediatr 1986; 109: 959-64.

18. Maciel RMB. Interpretações laboratoriais nas doenças da tiróide. In Setian N (ed): Endocrinologia pediátrica. São Paulo, Sarvier, 1989; 253-68.

19. Walfish PG. Screening for neonatal hypothyroidism. In Meites S (ed): Pediatric clinical chemistry: a survey of reference (normal) values, methods and instrumentation with commentaries. Washington DC, American Association of Clinical Chemists, 1981; 465-570.

20. Maciel RMB, Vieira J GH, Russo EMK et al. In Meirelles RMR, Machado A, Póvoa LC (eds): Clinical thyroidology. Amsterdam, Exerpta Medica, 1988; 145-8.

21. Fisher DA. Management of congenital hypothyroidism. J Clin Endocrinol Metab 1991; 72: 523-9.

22. Vieira J GH, Kunii IS, Nishida SK et al. Desenvolvimento e aplicação de um método imunofluorimétrico para a dosagem de tirotrofina humana (TSH) no soro e em sangue total colhido em papel de filtro. Arq Bras Endocrinol Metab 1992; 36: 7-12.

23. American Academy of Pediatrics and American Thyroid Association. Newborn screening for congenital hypothyroidism: recommended guidelines. Thyroid 1993; 257-63. 